\title{
Stroke Statistics in Korea: Part II Stroke Awareness and Acute Stroke Care, A Report from the Korean Stroke Society and Clinical Research Center For Stroke
}

\author{
Keun-Sik Hong, ${ }^{a}$ Oh Young Bang, ${ }^{b}$ Jong S. Kim, ${ }^{c}$ Ji Hoe Heo, ${ }^{d}$ Kyung-Ho Yu, ${ }^{e}$ Hee-Joon Bae, ${ }^{f}$ \\ Dong-Wha Kang, ${ }^{c}$ Jin Soo Lee, ${ }^{\mathrm{g}}$ Sun U. Kwon, ${ }^{\mathrm{c}}$ Chang Wan Oh, ${ }^{\mathrm{h}}$ Byung-Chul Lee, ${ }^{\mathrm{d}}$ Byung-Woo Yoon ${ }^{\mathrm{i}}$ \\ ${ }^{\mathrm{a}}$ Department of Neurology, Ilsan Paik Hospital, Inje University College of Medicine, Goyang; ${ }^{\mathrm{b}}$ Departments of Neurology, Samsung Medical Center, \\ Sungkyunkwan University School of Medicine, Seoul; 'Department of Neurology, University of Ulsan College of Medicine, Ulsan; ${ }^{\mathrm{d} D e p a r t m e n t ~ o f ~}$ \\ Neurology, Yonsei University College of Medicine, Seoul; ${ }^{\mathrm{D}}$ Department of Neurology, Hallym University College of Medicine, Aanyang; ${ }^{\mathrm{f}} \mathrm{Department}$ of \\ Neurology, Bundang Seoul National University College of Medicine, Seongnam; ${ }^{\mathrm{g} D e p a r t m e n t}$ of Neurology, Ajou University School of Medicine, Suwon; \\ ${ }^{\mathrm{h}}$ Department of Neurosurgery, Bundang Seoul National University College of Medicine, Seongnam; ${ }^{\mathrm{D}}$ Department of Neurology, Seoul National University \\ College of Medicine, Seoul, Korea
}

The aim of the current Part II of Stroke Statistics in Korea is to summarize nationally representative data on public awareness, pre-hospital delay, thrombolysis, and quality of acute stroke care in a single document. The public's knowledge of stroke definition, risk factors, warning signs, and act on stroke generally remains low. According to studies using openended questions, the correct definition of stroke was recognized in less than $50 \%$, hypertension as a stroke risk factor in less than $50 \%$, and other well-defined risk factors in less than $20 \%$. Among stroke warning signs, sudden paresis or numbness was best appreciated, with recognition rates ranging in 36.9-73.7\%, but other warning signs including speech disturbance were underappreciated. In addition, less than one third of subjects in a representative population survey were aware of thrombolysis and had knowledge of the appropriate act on stroke, calling emergency medical services (EMS). Despite EMS being an essential element in the stroke chain of survival and outcome improvement, EMS protocols for field stroke diagnosis and prehospital notification for potential stroke patients are not well established. According to the Assessment for Quality of Acute Stroke Care, the median onsetto-door time for patients arriving at the emergency room was 4 hours (mean, 17.3 hours) in 2010, which was not reduced compared to 2005. In contrast, the median door-to-needle time for intravenous tissue plasminogen activator (IV-TPA) treatment was 55.5 minutes (mean, 79.5 minutes) in 2010, shorter than the median time of 60.0 minutes (mean, 102.8 minutes) in 2008. Of patients with acute ischemic stroke, 7.9\% were treated with IV- TPA in 2010, an increase from the 4.6\% in 2005. Particularly, IV-TPA use for eligible patients substantially increased, from $21.7 \%$ in 2005 to $74.0 \%$ in 2010 . The proportion of hospitals equipped with a stroke unit has increased from $1.1 \%$ in 2005 to $19.4 \%$ in 2010. Performance, as measured by quality indicators, has steadily improved since 2005 , and the performance rates for most indicators were greater than $90 \%$ in 2010 except for early rehabilitation consideration (89.4\%) and IV-TPA use for eligible patients (74.0\%). In summary, the current report indicates a substantial improvement in in-hospital acute stroke care, but also emphasizes the need for enhancing public awareness and integrating the prehospital EMS system into acute stroke management. This report would be a valuable resource for understanding the current status and implementing initiatives to further improve public awareness of stroke and acute stroke care in Korea.
Correspondence: Byung-Woo Yoon Department of Neurology, Seoul National University Hospital, 101 Daehak-ro, Jongno-gu, Seoul 110-744, Korea

Tel: +82-2-2072-2875

Fax: +82-2-3672-7553

E-mail: bwyoon@snu.ac.kr

Received: April 8, 2013

Revised: May 4, 2013

Accepted: May 5, 2013

This work was supported by the a grant (A102065) from the Korea Health 21 REtD project, Ministry of Health, Wel-fare and Family Affairs, Republic of Korea.

The authors have no financial conflicts of interest.

Keywords Stroke; Statistics; Public awareness; Acute stroke; Care 
Every year in Korea, approximately 105,000 people experience a new or recurrent stroke and more than 26,000 die of stroke, indicating that every 5 minutes stroke attacks someone and every 20 minutes stroke kills someone. Encouragingly, stroke mortality has decreased by $28.3 \%$ in the first decade of the 21 st century, possibly due to improvements in risk factor control and acute stroke care. ${ }^{1}$ However, because of the rapid increase of the elderly population in Korea, ${ }^{2}$ the fastest among the Organization for Economic Cooperation and Development (OECD) countries, ${ }^{3}$ stroke incidence is projected to increase by 3.5-fold by $2030 .{ }^{4}$ To cope with the tremendous stroke burden in the decades to come, the acute stroke care needs to be provided in a more efficient way, in parallel with efforts to prevent stroke. Reperfusion therapy, stroke unit care, and other evidence-based acute stroke therapies must undoubtedly improve the functional outcome and quality of life of acute stroke victims and reduce the burden of stroke on society and patients' families.

To delineate the current status of the burden, public awareness, and acute management of stroke in Korea, the Korean Stroke Society (http://www.stroke.or.kr/) and the Clinical Research Center for Stroke (http://www.stroke-crc.or.kr/) decided to summarize a representative summary of stroke statistics. As a result, we published Stroke Statistics in Korea: Part I Epidemiology and Risk factors. ${ }^{1}$ The present article aimed to summarize data on public awareness, pre-hospital delay, the status of thrombolysis, and the quality of acute stroke care. We searched the most representative national data from reports of the Korean government or related organizations. When nationally representative data were not available, we searched and summarized data from published articles, recognizing the limitations. Along with the earlier report on epidemiology and risk factors for stroke, this summary will serve as a valuable resource for healthcare providers, researchers, policy makers, and the public who seek the best national data on public awareness and acute stroke care in Korea.

\section{Public awareness: stroke definition, risk factors, warning signs, and act on stroke}

Public awareness of stroke definition, risk factors, warning signs, and act on stroke is the important step in the chain of stroke survival and outcome improvement. To review the current status of public awareness in Korea, we systematically searched PubMed and KoreaMed with the terms "stroke" or "cerebrovascular" AND "awareness" or "recognition" or "knowledge" or "perspective" AND "Korea". Then, we selected populationbased studies with a sufficient sample size (at least 500). For pre-hospital delay and thrombolysis, we searched articles with the terms "stroke" or "cerebrovascular" AND "delay" or "alert" or "activation" or "computer" AND "hospital" AND "Korea", or with the terms "stroke" or "cerebrovascular" AND "119" or "EMS" or "paramedic" AND "Korea". For these issues, well-designed, small, hospital-based studies were also considered.

In studies of public awareness of stroke definition, only $44.8 \%$ of respondents in a study using open-ended question were able to correctly state that stroke is a vascular disease of the brain was, ${ }^{5}$ whereas the rates were $55.4-81.6 \%$ in studies using closeended questions..$^{6.8}$ Degenerative or aging process in the brain and convulsive disorder were the most common incorrect responses. In a recent survey sampling a nationwide representative population, people of middle age, and those with hypertension, hypercholesterolemia, and a family history of stroke were more likely to have correct knowledge of stroke definition. ${ }^{8}$

Several studies using open-ended questions have indicated that public knowledge of risk factors for stroke remains poor. ${ }^{7-11}$ Only about $50 \%$ of the public were able to identify at least 1 stroke risk factor (range, 48.1-56.4\%)(Figure 1). Among the risk factors, hypertension was the most commonly recognized risk factor, but awareness of this risk factor was generally low (range, 22.3-44.0\%). In all studies, smoking, a highly preventable risk factor, was less well recognized as a risk factor for stroke (range, $0.2-21.2 \%$ ), and fewer than $15 \%$ of respondents identified diabetes and hypercholesterolemia as risk factors (Figure 2). Knowledge of risk factors is lower in the Korean population than in the US population. ${ }^{12}$ In contrast, studies employing close-ended questions have resulted in higher percentages of respondents identifying risk factors (Figure 2). ${ }^{5-7,13}$ However, stress, an as yet unproven risk factor, was more often recognized as a risk factor (range, 59.7-87.8\%) than other well-documented and modifiable risk factors. Lower education level, older age, and incorrect knowledge of stroke definition were associated

Knowing $\geq 1$ risk factors (open-ended question)

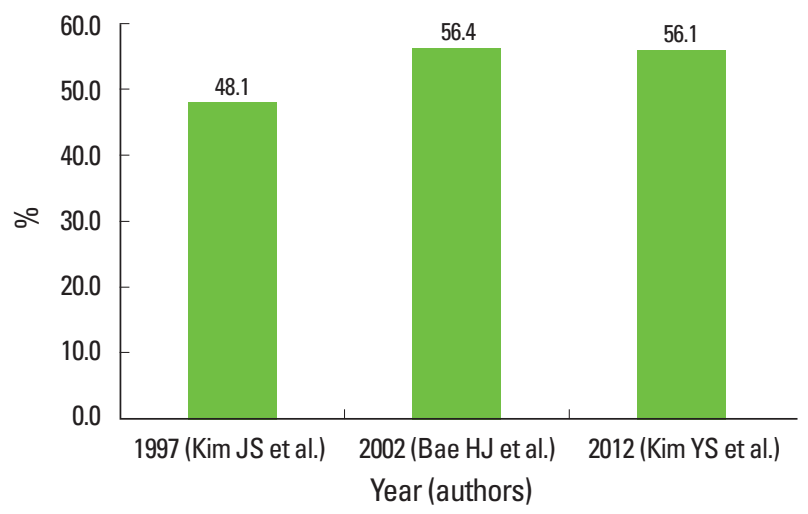

Figure 1. Public awareness of stroke risk factors: Knowing one or more risk factors. Source: Kim JS et al. ${ }^{9}$ Bae HJ et al. ${ }^{10}{ }^{10}$ Kim YS et al. ${ }^{8}$ 

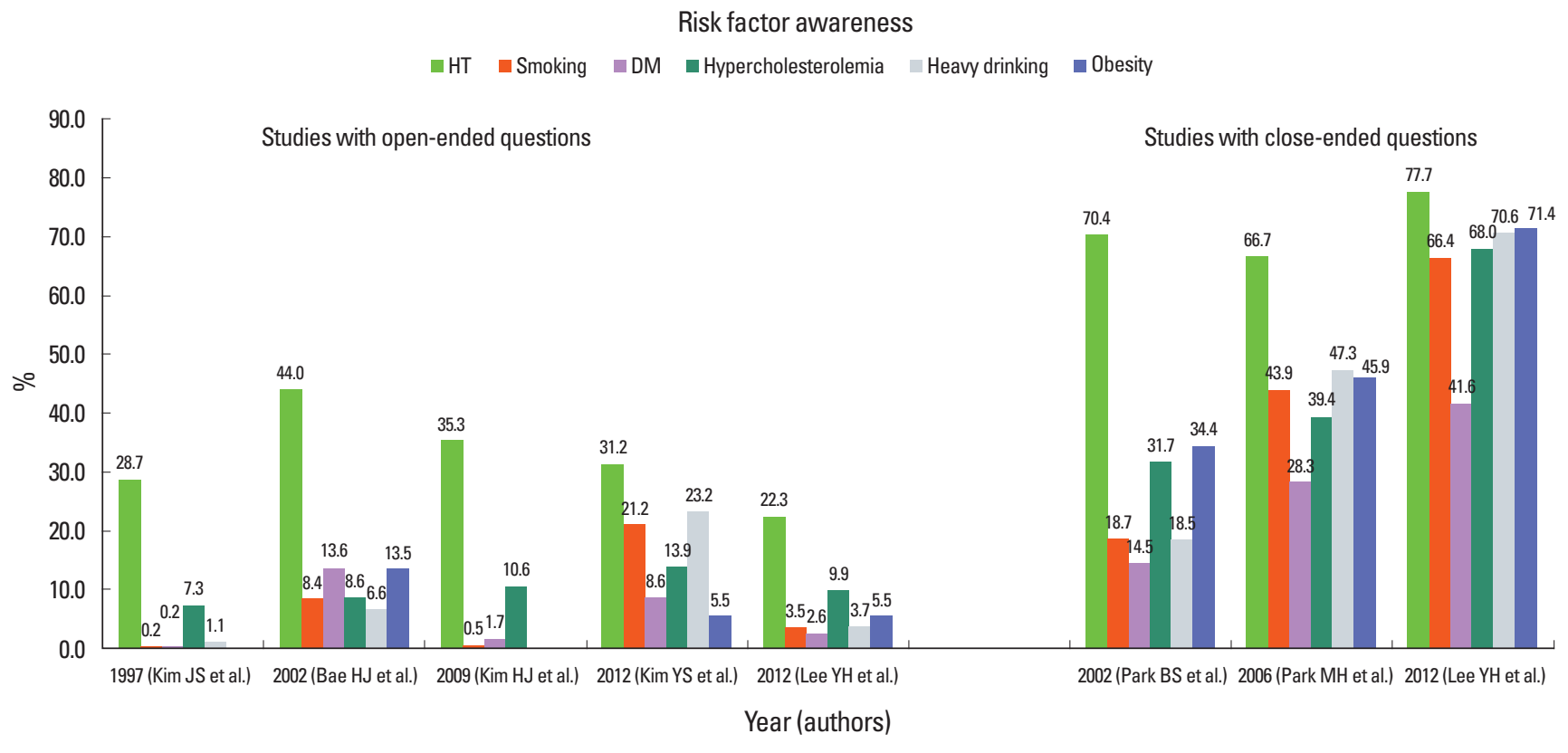

Figure 2. Public awareness of Individual risk factors. Source: Kim JS et al., ${ }^{9}$ Bae HJ et al., ${ }^{10}$ Kim HJ et al.,. ${ }^{11}$ Kim YS et al., ${ }^{8}$ Lee $Y H$ et al., ${ }^{7}$ Park BS et al., ${ }^{6}$ Park MH et al. ${ }^{5}$

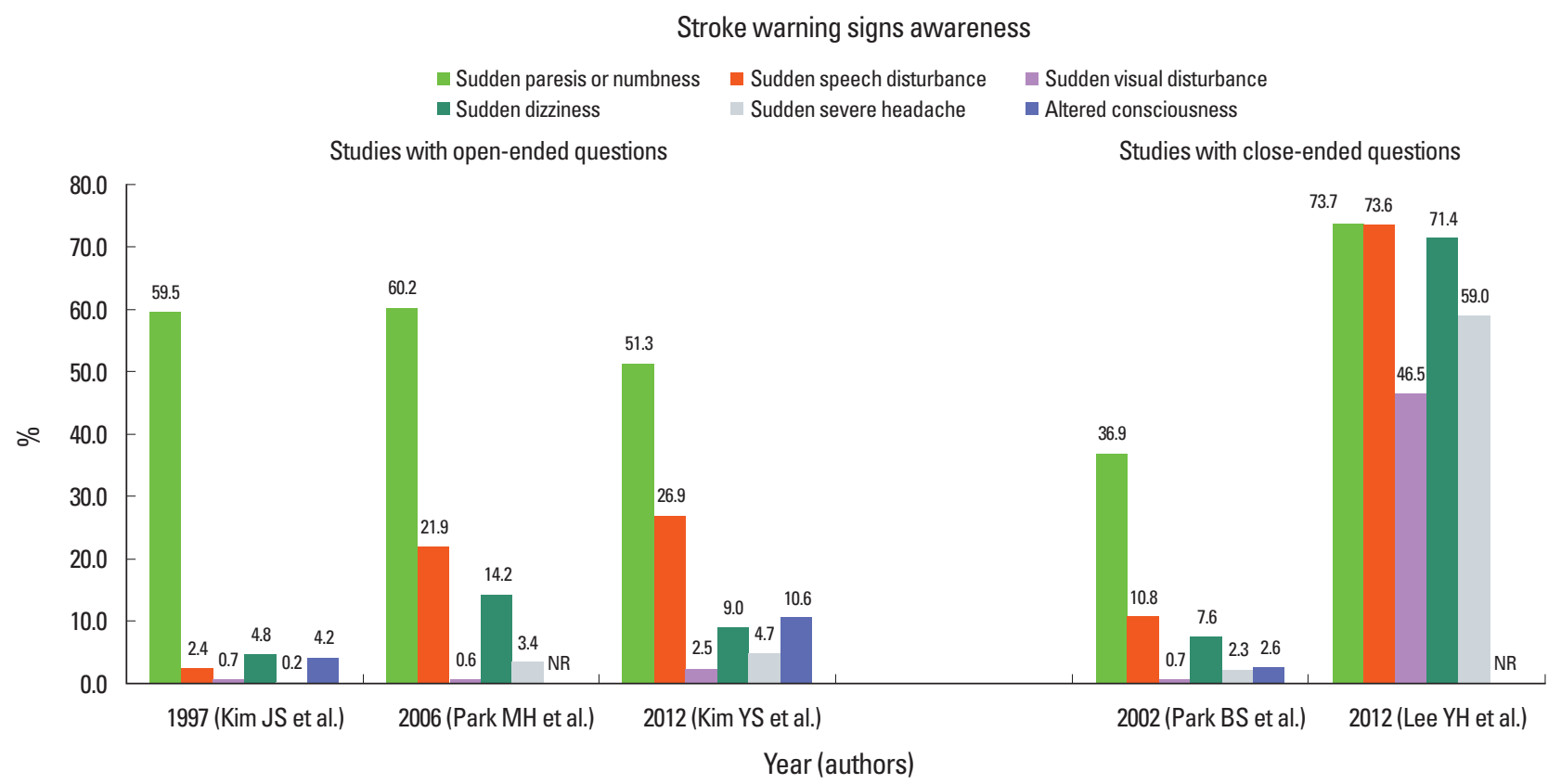

Figure 3. Public awareness of stroke warning signs. Source: Kim JS et al., ${ }^{9}$ Park MH et al., ${ }^{5}$ Kim YS et al., ${ }^{8}$ Park BS et al., ${ }^{6}$ Lee YH et al. ${ }^{7}$

with reduced risk factor awareness. ${ }^{5,7,8,10}$ In one study, current smokers had poorer awareness of risk factors. ${ }^{7}$ Interestingly, several studies found that people having stroke risk factors did not have a better knowledge of stroke risk factors than those without stroke risk factors. ${ }^{6,710}$ These findings suggest that physicians need to endeavor to further educate their patients on the goal of the management and control of their risk factors.

For stroke warning signs, studies questioning in an open-ended fashion showed that $60-75 \%$ of the Korean people were able to correctly identify one or more stroke warning signs. ${ }^{8,9}$ How- ever, another study showed that only $24.3 \%$ of respondents had knowledge of at least 2 stroke warning signs. ${ }^{5}$ Across all studies using open-ended or close-ended questions, sudden paresis or numbness was the most commonly recognized stroke symptom (range, 36.9-73.7\%) (Figure 3) ${ }^{5-9}$ Except for one study surveying an urban population using close-ended questions, ${ }^{7}$ the awareness of other stroke warning signs was generally low: speech disturbance was recognized by $2.4-26.9 \%$, visual disturbance by $0.6-2.5 \%$, dizziness or imbalance by $4.8-14.2 \%$, and sudden severe headache by $0.2-4.7 \%$. People with lower educa- 
tional level were less likely to have knowledge of stroke warning signs, ${ }^{5,78}$ and incorrect knowledge of stroke definition was also associated with reduced awareness of stroke warning signs. ${ }^{5,7}$

For act on stroke, a recent survey sampling a nationwide representative population showed that only $33 \%$ of respondents chose the proper action of calling emergency medical services (EMS) if they had a stroke warning sign. Compared with younger people, the middle-aged population was more likely to choose the appropriate act on stroke. ${ }^{8}$ However, $68.6 \%$ of urban people aged $\geq 50$ years and $79.0 \%$ of people voluntarily participating in a nationwide stroke educational program performed by the Korean Stroke Society responded that they would call EMS.,10 Therefore, people who are interested in stroke prevention and treatment are more likely to have a better knowledge of act on stroke.

For thrombolysis, $31 \%$ of the general public who were surveyed in a nationwide representative sampling had some knowledge of thrombolysis, ${ }^{8}$ and the percentage was greater than the $19 \%$ reported in a US study. ${ }^{12}$ However, in a multicenter study conducted in the Seoul metropolitan area enrolling patients with acute ischemic stroke within 48 hours of onset, only $18 \%$ of patients were aware of thrombolysis. Of them, $87 \%$ knew that thrombolysis should be performed as soon as possible or within 3 hours. In this study, patient's or bystander's knowledge of stroke warning signs reduced the pre-hospital delay, and people with higher educational level were more likely to have knowledge of thrombolysis. ${ }^{14}$

Across all studies, media broadcasting (television or radio) was the most common source of information about stroke (Figure 4) ${ }^{5.9}$ In most studies, laypersons such as family members, friends, and neighbors were more often cited as sources of information than were experts; this phenomenon has the potential to drive public knowledge away from science-based principles. ${ }^{5-7,9}$ Reflecting the advancement of information technology, a recent survey showed that the internet was ranked second among the sources of information for people aged 20-39 years. ${ }^{8}$ Therefore, academic societies need to provide scientific knowledge in more appropriate and understandable ways.

Public knowledge of stroke has been investigated in different populations and with different methods. Accordingly, the current summary provided an overview of public awareness across diverse populations, but could not adequately assess temporal trends. However, studies using almost identical methods have shown that the public's recognition of hypertension and hyperlipidemia as risk factors for stroke has improved. ${ }^{9,11}$

\section{Pre-hospital delay}

According to the Report of Assessment for Quality of Acute Stroke Care in Korea 2010 which analyzed data from patients admitted via the emergency room (ER) within 7 days of onset, the median interval from onset to ER admission was 243 minutes(interquartile range [IQR], 82-894 minutes) and the mean interval was $824 \pm 1,437$ minutes. The prehospital delay was greater in patients with ischemic stroke than in those with

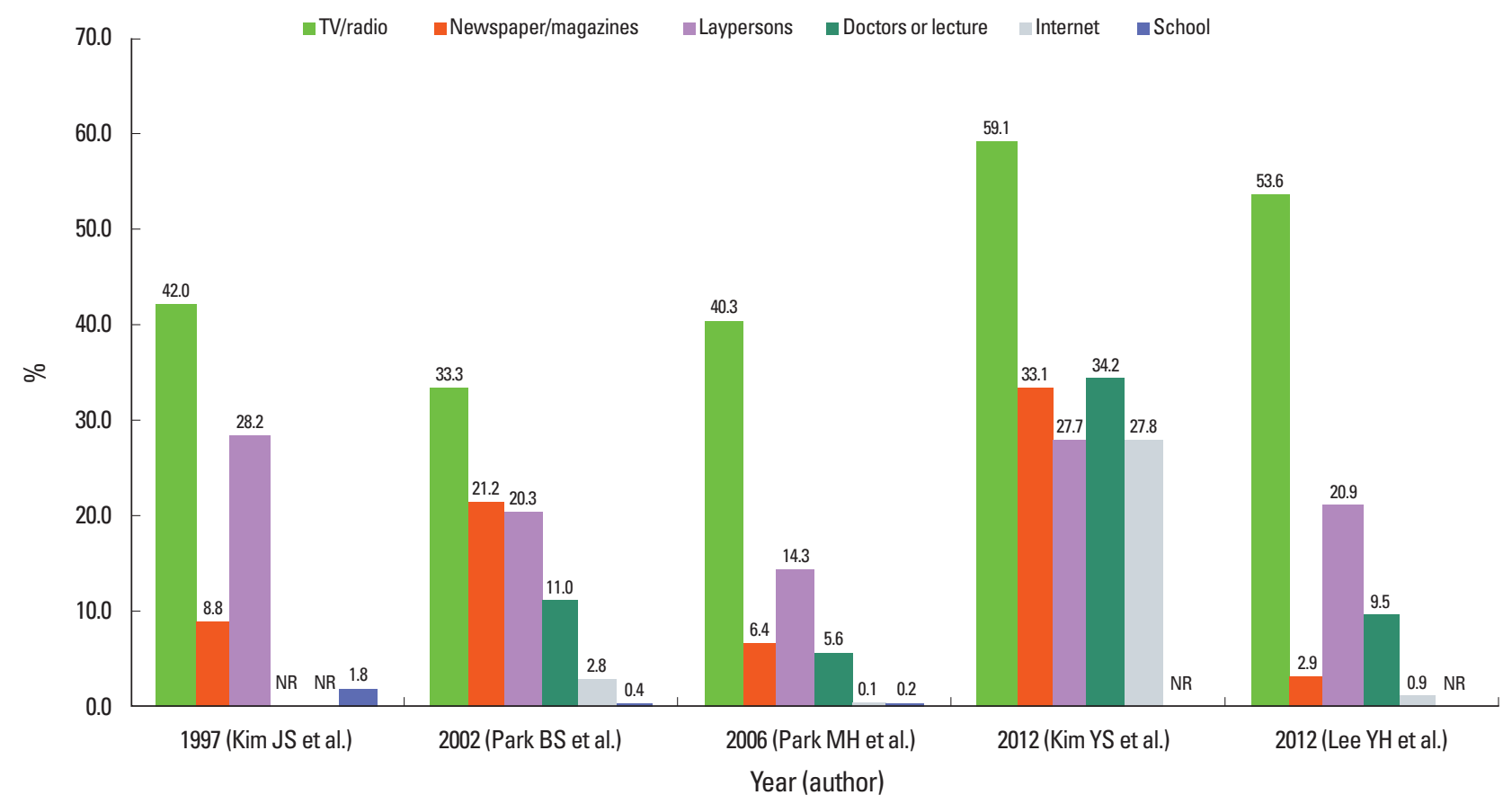

Figure 4. Information source of stroke. Source: Kim JS et al., ${ }^{9}$ Park BS et al., ${ }^{6}$ Park MH et al., ${ }^{5}$ Kim YS et al., ${ }^{8}$ Lee YH et al. ${ }^{7}$ 
hemorrhagic stroke (median [IQR], 350 [107-1,179] minutes vs 141 [56-387] minutes; mean \pm standard deviation, $959 \pm$ 1,504 minutes vs $554 \pm 1,250$ minutes). Compared with data from 2005 and 2008, the prehospital delay was greater in 2010 (Figure 5) ${ }^{15-17}$ For any stroke, the proportions of patients arriving within 1 hour, 2 hours, 3 hours, and 6 hours were 19.4\%, $33.7 \%, 43.3 \%$, and $58.4 \%$ in 2010 , respectively; these values were lower than those from 2008. For ischemic stroke, the pro-

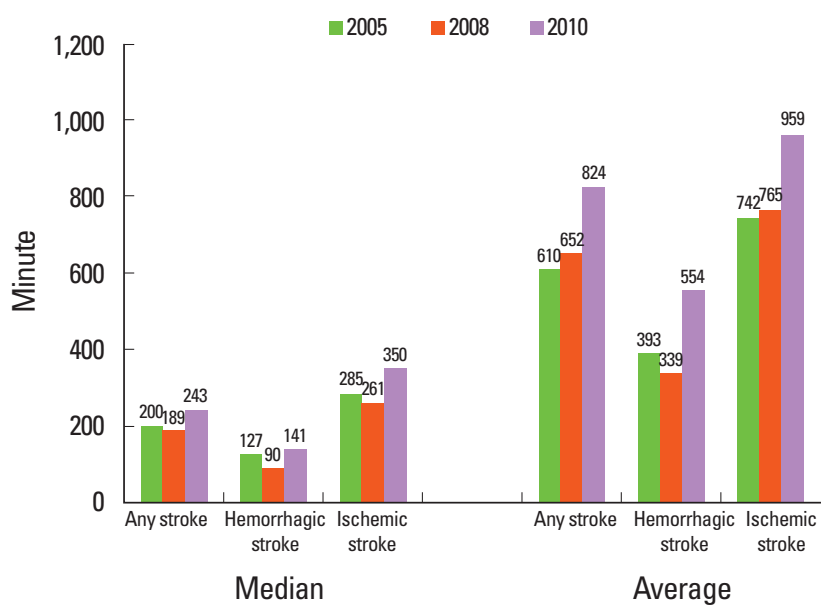

Figure 5. Interval from onset to ER. Source: Korean Health Insurance Review \& Assessment Service. Report of Assessment for Quality of Acute Stroke Care in Korea 2005-2010. ${ }^{15-17}$ portions of patients arriving within 2 hours and 3 hours, who could be eligible for the $<3$ hours and $<4.5$ hours intravenous tissue plasminogen activator (IV-TPA) time windows, were $27.8 \%$ and $36.3 \%$ in 2010; these values were lower than those from 2008 (Figure 6). ${ }^{16,17}$ However, a nationwide hospital-based stroke registry study showed contrary findings: the proportion of patients with ischemic stroke admitted within 3 hours of symptom onset increased from $20.2 \%$ in 2002 to $28.6 \%$ in 2010 ( $\mathrm{p}$ for trends $<0.001$ ) (Figure 7). ${ }^{18}$

The Report of Assessment for Quality of Acute Stroke Care in Korea 2010 showed that patients transported by EMS, compared with those who were not, had a shorter prehospital delay (median [IQR], 162 [50-526] minutes vs 468 [140-1,425] minutes; mean \pm standard deviation, $603 \pm 1,226$ minutes vs $1,125 \pm$ 1,641 minutes) and were more likely to arrive within 3 hours (Figures 8,9). In a multicenter study of 14 hospitals in the Seoul metropolitan area enrolling patients with ischemic stroke within 48 hours, the utilization of EMS was independently associated with arrival within 3 hours of stroke onset (53.6\% vs $29.7 \%) .{ }^{14}$ Awareness of stroke warning signs, knowledge of thrombolysis, and severe stroke were other independent predictors for early arrival, after adjusting for covariates. ${ }^{14}$ Several hospital-based studies also demonstrated that EMS use was associated with earlier arrival. ${ }^{19-21}$

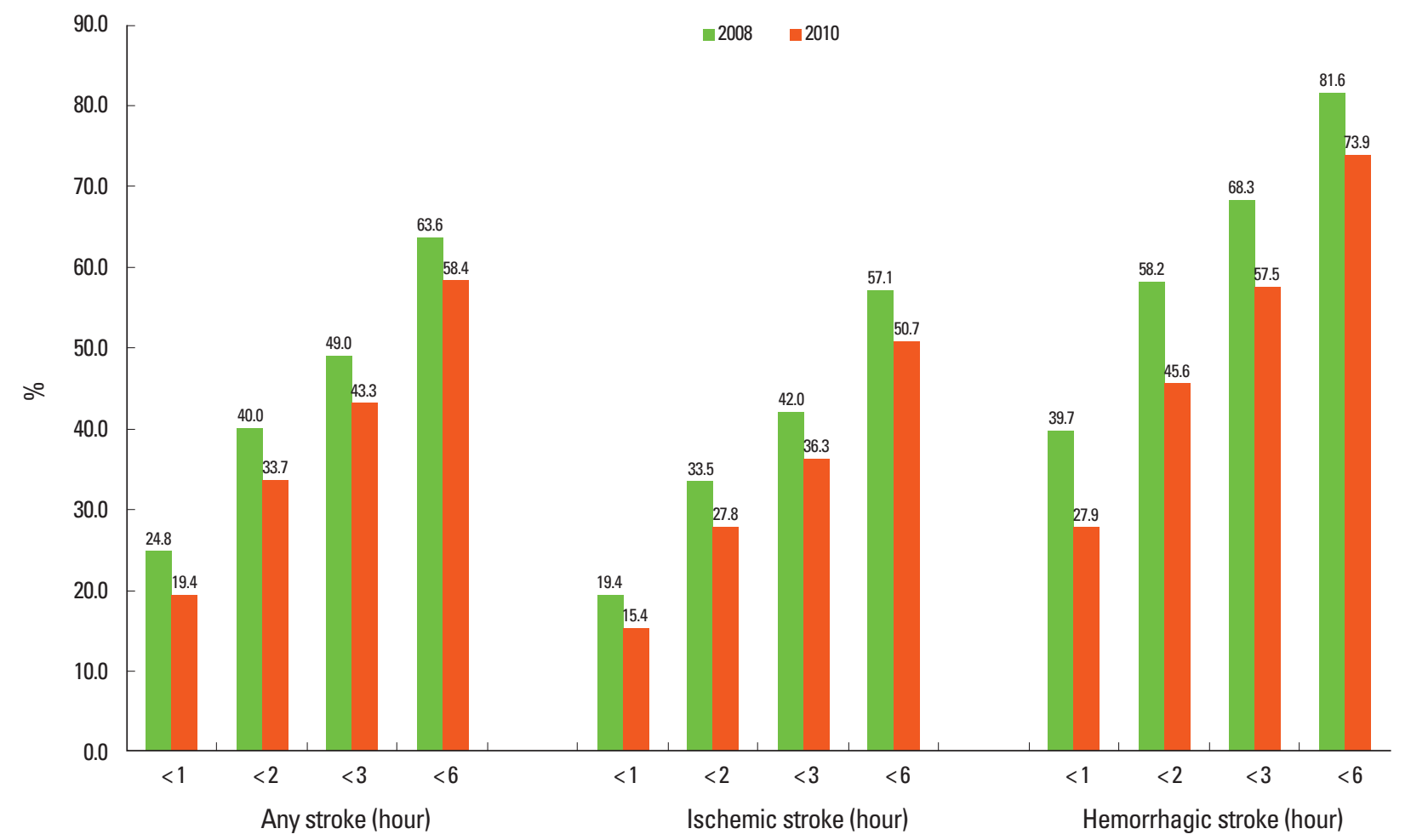

Figure 6. Proportion of arrival by time. Source: Korean Health Insurance Review \& Assessment Service. Report of Assessment for Quality of Acute Stroke Care in Korea 2008-2010. ${ }^{16,17}$ 


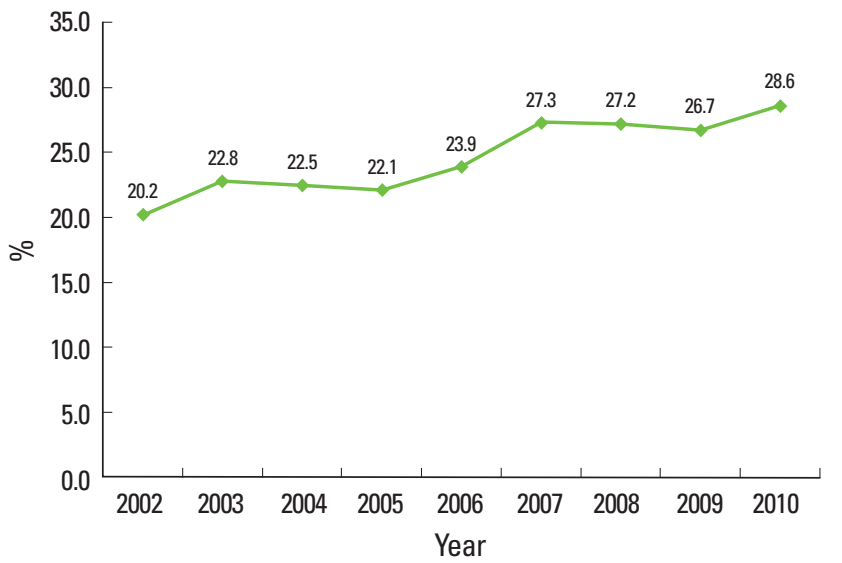

Figure 7. Arrival within 3 hours from onset in patients with ischemic stroke. Source: Jung KH et al..$^{18}$

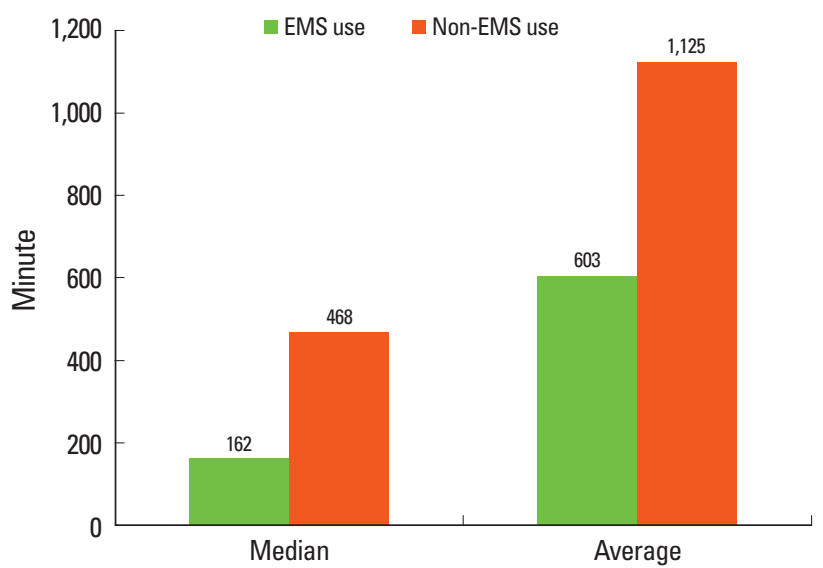

Figure 8. Interval from onset to ER by transportation. Source: Korean Health Insurance Review \& Assessment Service. Report of Assessment for Quality of Acute Stroke Care in Korea 2010. ${ }^{17}$

\section{Prehospital notification from EMS}

No nationwide representative data are available on the rates of prehospital notification by EMS of a potential stroke. However, it is generally assumed that the rate of prehospital notification is quite low in Korea. Even for a center with a formal EMS prehospital notification system, prehospital notification was performed for fewer than one-third of patients treated with IVTPA. $^{22}$ In that study, IV-TPA treatment rate increased after the implementation of the prehospital notification system, and door-to-imaging and door-to-needle intervals were significantly shorter for patients with prehospital notification than for those without prehospital notification.

A study performed in the Busan area in 2010 showed that the mean interval between a patient's or relative's call to EMS and hospital arrival was 26 minutes; this indicates fast deployment and transportation of EMS personnel. Stroke diagnosis by EMS personnel was correct in only $42.5 \%$ of cases. However, the

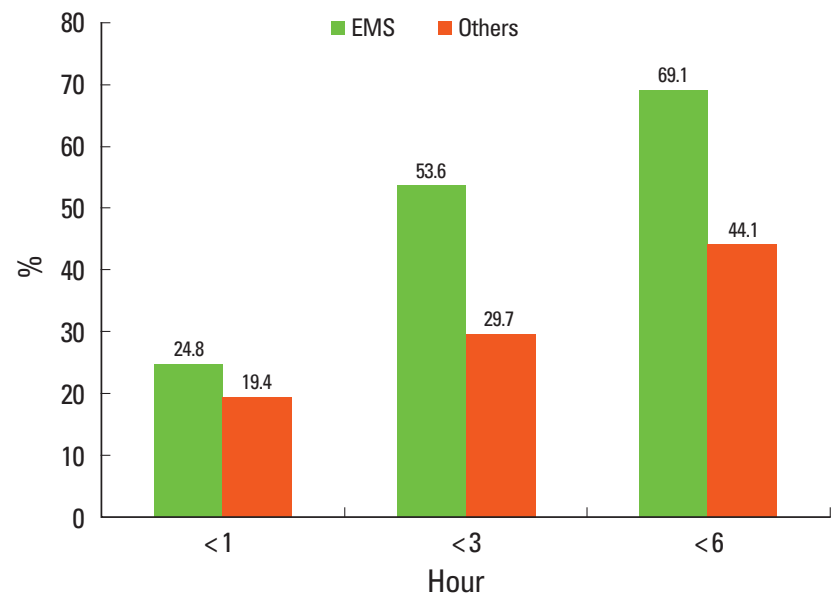

Figure 9. Proportion of arrival by time and transportation. Source: Korean Health Insurance Review \& Assessment Service. Report of Assessment for Quality of Acute Stroke Care in Korea 2010.17

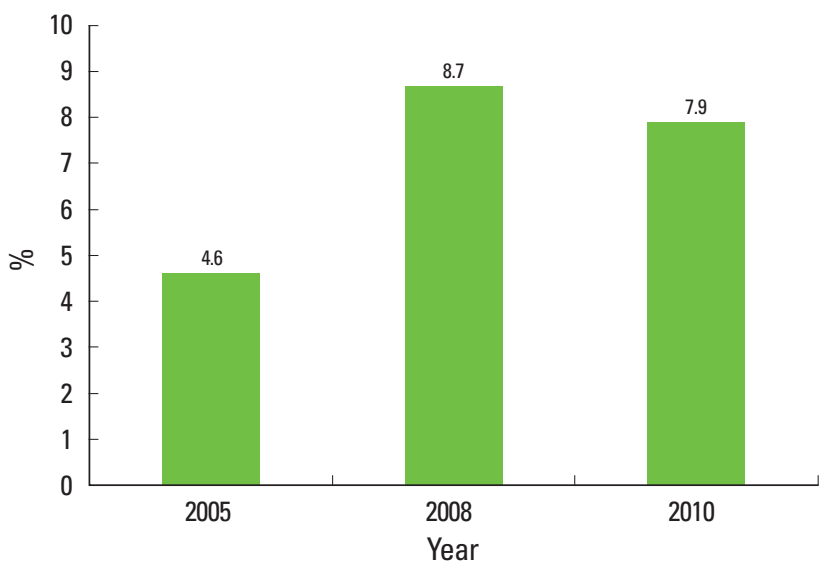

Figure 10. IV-TPA treatment rate among ischemic stroke by year. Source: Korean Health Insurance Review \& Assessment Service. Report of Assessment for Quality of Acute Stroke Care in Korea 2005-2010. ${ }^{15-17}$

study did not use a formal stroke screening test, such as Los Angeles Prehospital Stroke Screen or Cincinnati Prehosptial Stroke Scale, ${ }^{23,24}$ which have proved effective in diagnosing stroke patients in the field.

Since acute stroke therapies are critically time-dependent and EMS is an essential element in the chain of stroke survival and outcome improvement, nationwide standardized education and stroke care protocols for EMS need to be implemented.

\section{Thrombolysis}

The Report of Assessment for Quality of Acute Stroke Care in Korea 2010 analyzed data from 7,864 patients with acute stroke admitted to 201 hospitals via the ER within 7 days of stroke onset for a 3-month period in 2010: 5,264 (66.0\%) were ischemic stroke patients and 2,600 (33.1\%) were hemorrhagic stroke patients. Of 5,264 patients with ischemic stroke, 7.9\% 

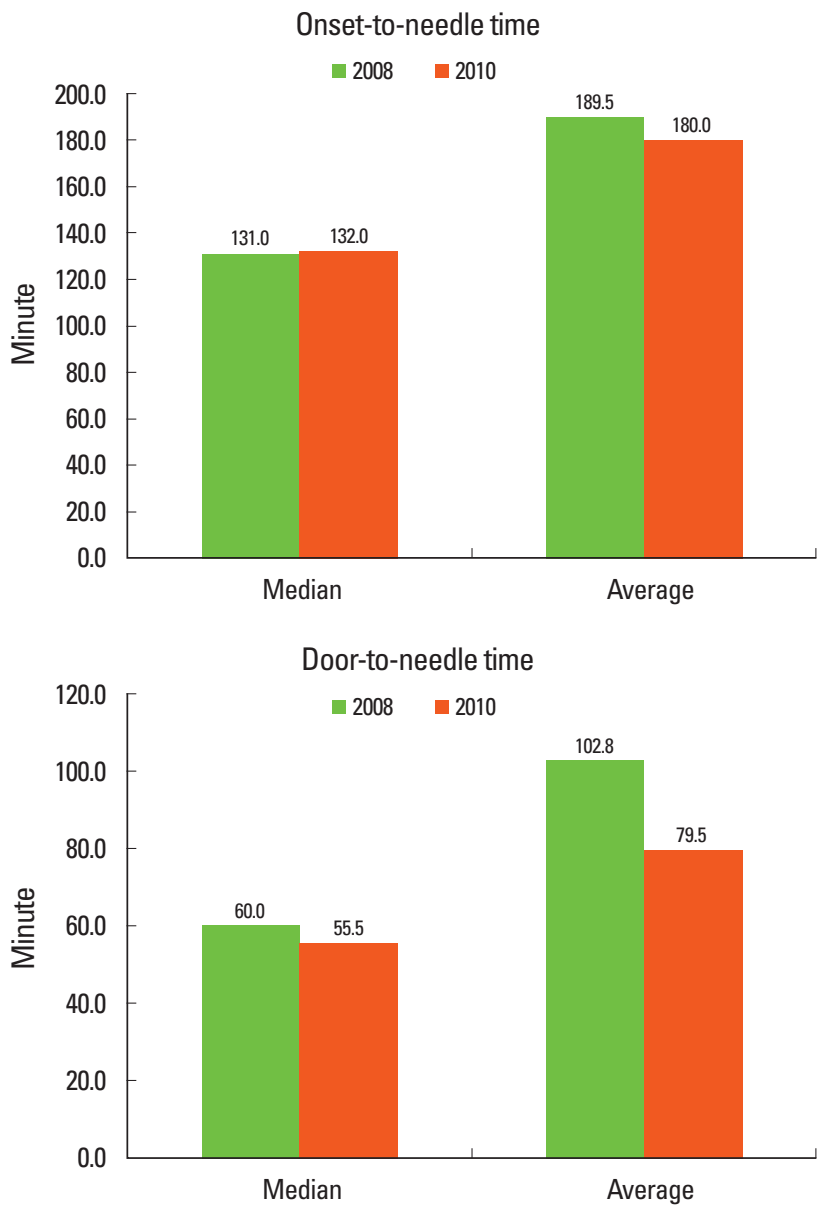

Figure 11. Onset-to-needle and door-to-needle time for IV-TPA treatment. Source: Korean Health Insurance Review \& Assessment Service. Report of Assessment for Quality of Acute Stroke Care in Korea 2008-2010. ${ }^{16,17}$

were treated with IV-TPA. This rate was higher than the $4.6 \%$ reported for 2005, but lower than the $8.7 \%$ reported for 2008 (Figure 10). ${ }^{15-17}$ The most common reason for the exclusion of IV-TPA treatment in 2010 was arrival after 3 hours (73.7\%), followed by minor or rapidly improving symptoms (15.5\%), risk of intracranial or extracranial bleeding (1.9\%), refusal by patients or surrogates $(0.8 \%)$, receipt of prior IV-TPA treatment in another hospital $(0.8 \%)$, possibility of diagnosis other than stroke $(0.6 \%)$, bleeding tendency in laboratory findings $(0.5 \%)$, uncontrolled hypertension (0.4\%), and others (5.8\%). Among 1,018 patients arriving within 2 hours of onset, IV-TPA was considered for 93.5\% in 2010; this figure is slightly higher than the 92.2\% reported for 2008. In 2010, the rate of IV-TPA treatment in these patients was $40.9 \%$, which was greater than the $36.7 \%$ reported for 2008. The reasons for treatment exclusion in patients presenting within 2 hours of onset were not reported.

Another nationwide hospital-based stroke registry study that enrolled all patients (admission via ER and outpatient clinic) showed that the thrombolysis rate has increased in Korea over

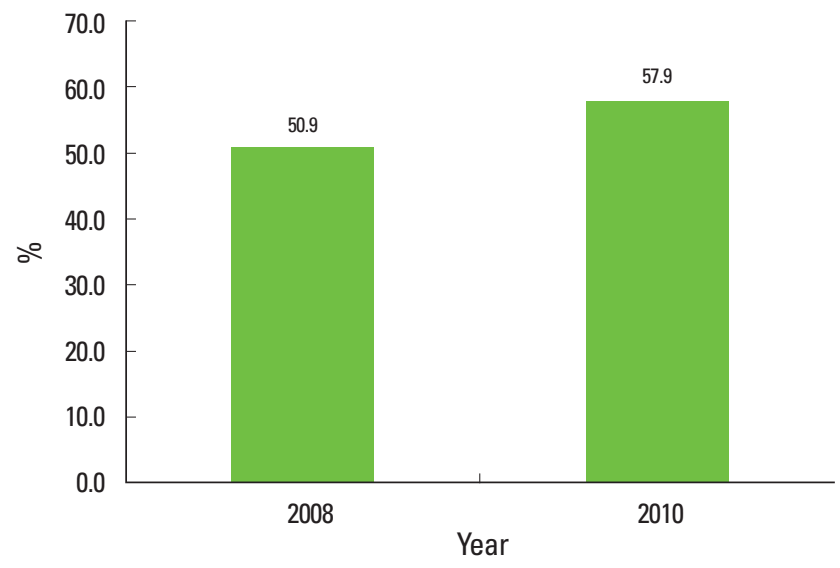

Figure 12. IV-TPA treatment rate within 1 hour after ER arrival. Source: Korean Health Insurance Review \& Assessment Service. Report of Assessment for Quality of Acute Stroke Care in Korea 2008-2010.16,17

the last decade. ${ }^{18}$ IV-TPA use has increased from $4.4 \%$ in 2002 to $6.0 \%$ in 2010 , and any reperfusion therapy (intravenous and/ or intra-arterial thrombolysis) has increased from $5.3 \%$ in 2002 to $7.0 \%$ in 2010 .

According to data from the Report of Assessment for Quality of Acute Stroke Care in Korea 2010, among patients treated with IV-TPA, treatment was initiated within 1 hour of stroke onset in 3.6\%, within 2 hours in 40.6\%, and within 3 hours in $84.9 \%$. The median interval from onset to treatment was 132.0 (IQR, 102.0-165.0) minutes, and the mean interval was 180.0 minutes; these figures were comparable to those for 2008 (median [IQR], 131.0 [100.0-169.0]; mean, 189.5). As compared to data from the 2008 Report of Assessment for Quality of Acute Stroke Care in Korea, the door-to-needle time substantially improved in 2010. Median door-to-needle time reduced from 60.0 (IQR, 46.0-83.0) minutes to 55.5 (41.0-80.0) minutes, and the mean door-to-needle time reduced from $102.8 \pm$ 404.6 minutes to $79.5 \pm 139.9$ minutes (Figure 11). The proportion of treatment administered within 1 hour of arrival increased by an absolute $7 \%$ (from $50.9 \%$ in 2008 to $57.9 \%$ in 2010) (Figure 12). ${ }^{15-17}$ These rates were substantially higher than the $26.6 \%$ reported for hospitals participating in the Get With the Guidelines-Stroke Program in the United States. ${ }^{25}$ In a multicenter study implementing acute stroke code program that activates acute stroke teams using information technology and a computerized physician order entry system, the intervals from door to computed tomography (CT) scan, door to accessibility of complete blood counts result, and door to accessibility of prothrombin time results reduced by 7.7 minutes, $5.6 \mathrm{~min}$ utes, and 26.8 minutes, respectively, compared with intervals before the program implementation $(P<0.001$ for all $) .{ }^{26}$ In a single-center study, patients who arrived earlier had longer inhospital delays for IV-TPA treatment before the program imple- 
Total population of South Korea, 51,003,843 (March 2013)

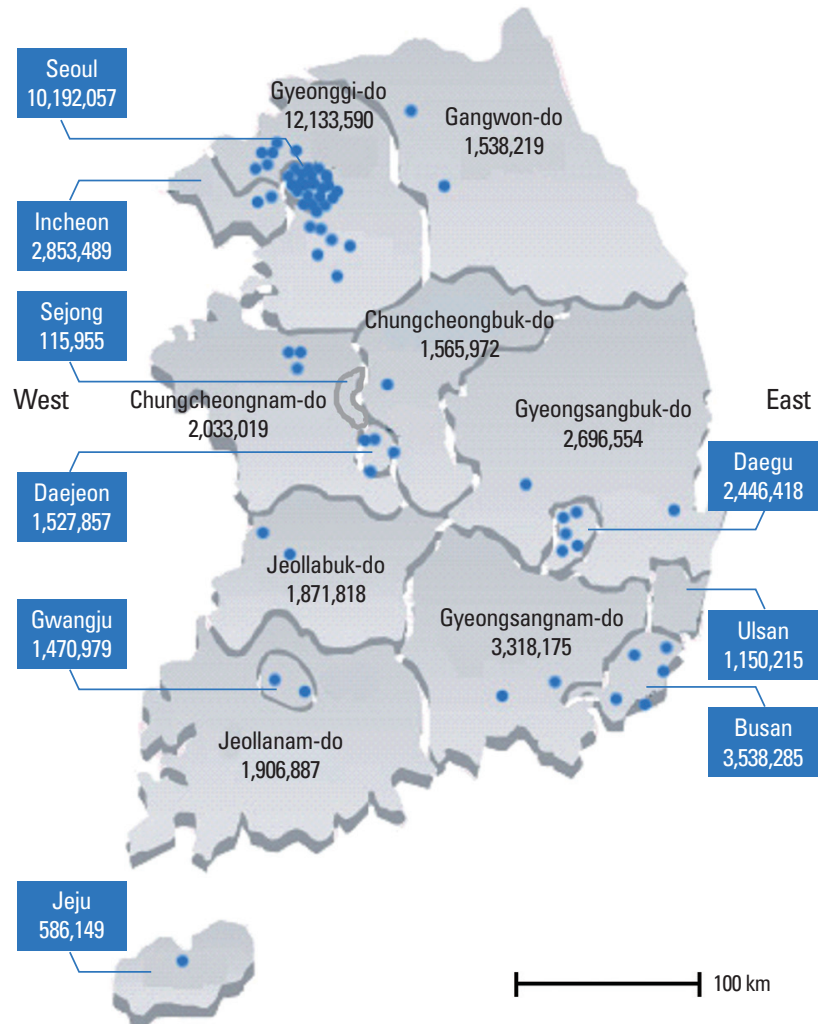

Figure 13. The distribution of 67 neurology training hospitals capable of providing IV-TPA treatment 24/7/365 and the population in each region. Source: Choi HY et al. ${ }^{28}$

mentation. However, the acute stroke code program abolished the inverse relationship between the onset-to-door and doorto-needle intervals. ${ }^{27}$

A nationwide survey of hospitals capable of providing IVTPA treatment has not yet been performed. However, in a survey of 67 neurology training hospitals (representing $78.0 \%$ of all neurology training hospitals), all participating centers responded that they were capable of providing IV-TPA treatment 24 hours a day, 7 days a week, 365 days a year (24/7/365). The distribution of centers and the populations in each region are presented in Figure 13. In addition, intra-arterial thrombolysis was available $24 / 7 / 365$ in 50 hospitals ( $74.6 \%$ of participating centers). ${ }^{28}$

\section{Stroke unit and other resources}

Since 2002 when the first stroke unit was established in Korea, the number of hospitals equipped with a stroke unit has increased. According to the Report of Assessment for Quality of Acute Stroke Care in Korea, the proportion of hospitals with a stroke unit has increased from $1.1 \%$ in 2005 to $19.4 \%$ in 2010 (Figure 14). In this assessment, a stroke unit was defined as an

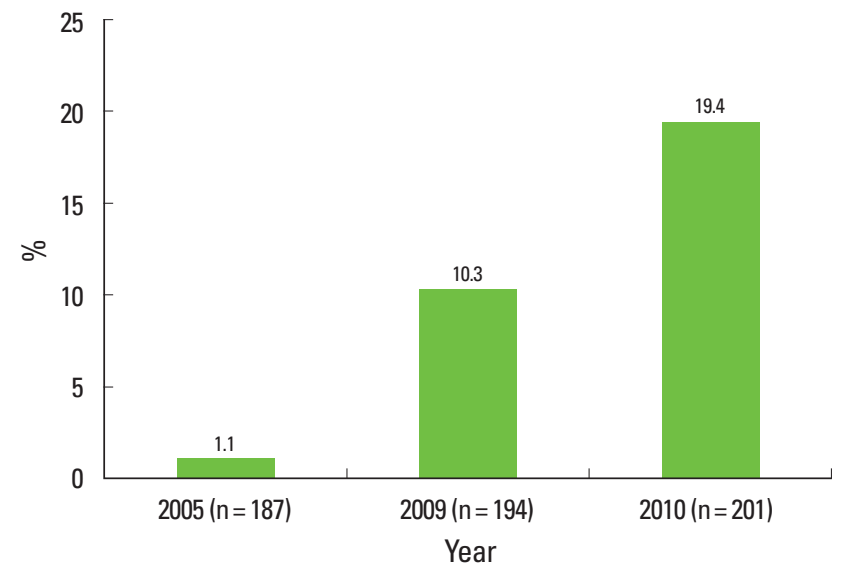

Figure 14. Hospitals with a stroke unit. Source: Korean Health Insurance Review \& Assessment Service. Report of Assessment for Quality of Acute Stroke Care in Korea 2005-2010. .5-17 $^{-17}$

independent space where dedicated stroke nursing staffs were deployed, cardiac and oxygen monitoring systems were available, and neurological status was assessed using a validated stroke scale.

Another study surveying 67 neurology training hospitals (representing $78.0 \%$ of all training hospitals) showed that $43.3 \%$ of the participating hospitals had a stroke unit in $2010 .{ }^{28}$ The number of hospitals with a stroke unit has increased substantially between 2007 and 2010. For all participating hospitals with and without a stroke unit, CT and CT angiography were available 24/7/365. Carotid duplex sonography, transcranial Doppler, conventional catheter angiography, and magnetic resonance angiography were also available in almost all hospitals, and $73.1 \%$ of hospitals had an magnetic resonance imaging accessibility $24 / 7 / 365$. All hospitals were capable of IV-TPA treatment and 50 hospitals (74.6\%) could perform intra-arterial reperfusion therapy at any time. The facilities for and capability of providing acute reperfusion therapies did not differ between hospitals with and without a stroke unit. However, hospitals with a stroke unit were more likely to have stroke coordinators, general management protocols, and stroke team educational programs than those without a stroke unit.

Currently, the Korean government does not reimburse stroke unit care. Given the proven benefit of and the additional human and structural resource allocation for stroke unit care, reimbursement should be ensured to further implement stroke unit care.

\section{Quality of acute stroke care}

The Health Insurance Review \& Assessment Service (HIRA) has assessed the quality of acute stroke care since $2005 .{ }^{15-17} \mathrm{In}$ 2010, 13 indicators (one for structure and 12 for process) were assessed, including the new indicators of brain imaging within 1 
Table 1. Quality indicators for acute stroke care in 2010

\begin{tabular}{|c|c|c|}
\hline Domain & Item & Indicator \\
\hline Structure & Treatment ability & Organization of specialist personnel (Specialists of neurology, neurosurgery, and rehabilitation medicine) \\
\hline \multirow{7}{*}{ Process: Acute stroke (I60-163) } & Patient status assessment & Documentation rate of smoking history (doctor's record) \\
\hline & \& management & Neurological examination rate \\
\hline & & (Category-consciousness, motor and sensory functions, cranial nerve exam, reflex function) \\
\hline & & Dysphagia examination rate (within 2 days) \\
\hline & Initial diagnosis & Brain imaging rate (within 24 hours) \\
\hline & & Brain imaging rate (within 1 hour)* \\
\hline & Initial treatment & Consideration rate of early rehabilitation (within 3 days) ${ }^{*}$ \\
\hline \multirow[t]{6}{*}{ Process: ischemic stroke (I63) } & Initial diagnosis & Lipid profile test rate (including the test within 30 days before admission) \\
\hline & Initial treatment & Consideration rate of IV-TPA administration \\
\hline & & IV-TPA administration rate* \\
\hline & & Antithrombotics administration rate (within 48 hours) \\
\hline & Secondary prevention & Antithrombotics prescription rate of at discharge \\
\hline & & Anticoagulants prescription rate at discharge (atrial fibrillation) \\
\hline
\end{tabular}

*Indicators added to assessment in 2010.

IV-TPA, intravenous tissue plasminogen activator.

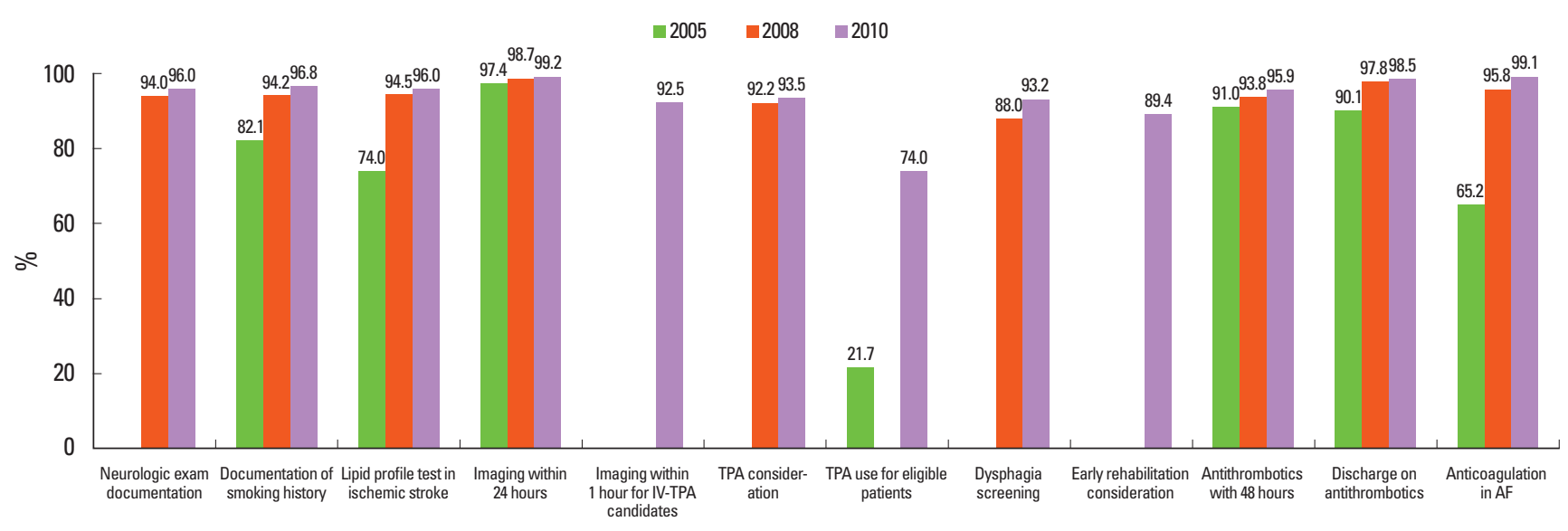

Figure 15. Quality indicators. Source: Korean Health Insurance Review \& Assessment Service. Comprehensive Quality Report of National Health Insurance 2010.17

hour for potential thrombolysis candidates, consideration of early rehabilitation within 3 days, and TPA administration rates in eligible patients (Table 1).

In 2010, the assessment was performed for 201 institutions (44 tertiary hospitals and 157 general hospitals) that claimed 10 or more acute stroke admissions via the ER (main disease with ICD 10 diagnostic code of I60-I63, admission within 7 days of onset) during the period from January to March 2010. For institutions that has fewer than 100 acute stroke inpatients, a complete survey was conducted, whereas institutions with 100 or more cases were assessed by a random sample of 100 cases. The overall performance rate for each indicator in the report was calculated from the data from institutions with $\geq 5$ denominator cases for each indicator. ${ }^{29}$

In 2010, physicians of neurology, neurosurgery, and rehabilitation with stroke expertise were all available in 128 (63.7\%) hospitals, an increase from the 112 (57.7\%) hospitals reported for $2008 .^{17}$
Performances in all indicators have steadily improved since 2005 (Figure 15)..$^{15-17,29}$ The mean performance rates for neurologic examination documentation, assessment of smoking history, lipid profile assessment, neuroimaging within 24 hours, antithrombotics within 48 hours, discharge on antithrombotics, and anticoagulation in atrial fibrillation were greater than $95 \%$ in 2010. The performance rates for dysphagia screening and consideration of IV-TPA were $93.2 \%$ and $93.5 \%$, respectively. The magnitude of improvement was greatest for anticoagulation in atrial fibrillation (from $65.2 \%$ in 2005 to $99.1 \%$ in 2010) followed by lipid profile assessment (from $74.0 \%$ in 2005 to $96.0 \%$ in 2010).

The performance rates for new quality indicators were lower than those of previously assessed indicators. However, the performance rates were generally high: $92.5 \%$ for brain imaging within 1 hour for potential thrombolysis candidates, $89.4 \%$ for consideration of early rehabilitation within 3 days, and $74.0 \%$ for IV-TPA administration in eligible patients. Particularly, the rate of IV-TPA administration for eligible patients (74.0\%) substan- 
tially improved by an absolute increase of $52.3 \%$, as compared to the $21.7 \%$ in 2005 (it was not an assessment indicator in 2005) (Figure 15).

\section{Summary}

The current report shows that the public's knowledge of stroke definition, risk factors, warning signs, and act on stroke remains low. In-hospital critical pathways for acute stroke management have substantially improved, but the prehospital EMS system is not well integrated into acute stroke management. Overall quality of acute stroke care is generally high, but the availability of stroke unit care should be more widely implemented in Korea.

\section{References}

1. Hong KS, Bang OY, Kang DW, Yu KH, Bae HJ, Lee JS, et al. Stroke Statistics in Korea: Part I. Epidemiology and Risk Factors: A Report from the Korean Stroke Society and Clinical Research Center for Stroke. J Stroke 2013;15:2-20.

2. Korean Statistical Information Service (KOSIS). Population Projections and Summary Indicator for Korea (Population items). http://kosis.kr/eng/database/database_001000.jsp?li stid=A\&subtitle=Population/Household. Accessed July 18, 2012.

3. Organization for Economic Cooperation and Development. OECD Economic Surveys: Korea 2010. OECD Web site. http://www.oecd.org/dataoecd/14/34/45432048.pdf. Accessed April 2011.

4. Bae HJ. Epidemiology of Stroke: 2006 Update. Korean J Stroke 2007;9:5-10.

5. Park MH, Jo SA, Jo I, Kim E, Eun SY, Han C, et al. No difference in stroke knowledge between Korean adherents to traditional and western medicine - the AGE study: an epidemiological study. BMC Public Health 2006;6:153.

6. Park BS, Lee JM, Koh SB, Kim BJ, Park MK, Park KW, et al. Ansan Citizen's Knowledge of Stroke. J Korean Neurol Assoc 2002;20:339-345.

7. Lee YH, Shin MH, Kweon SS, Choi JS, Park MS, Cho KH, et al. Awareness of Stroke Warning Signs and Risk Factors: Result of a 2010 Community Survey in Gwangju Metropolitan City. J Korean Neurol Assoc 2012;30:26-32.

8. Kim YS, Park SS, Bae HJ, Heo JH, Kwon SU, Lee BC, et al. Public awareness of stroke in Korea: a population-based national survey. Stroke 2012;43:1146-1149.

9. Kim JS, Yoon SS. Perspectives of stroke in persons living in Seoul, South Korea. Stroke 1997;28:1165-1169.
10. Bae HJ, Yoo KM, Yoon BW, Kim J, Kim JY, Kim EG, et al. Stroke Awareness in Korea: The results of survey in the second stroke prevention campaign. J Korean Neurol Assoc 2002;20: 110-117.

11. Kim HJ, Kim JS. Perspectives of stroke in South Korea: A survey of 1,503 subjects (abstract in the 28th Annual Meeting of the Korean Neurological Association). J Korean Neurol Assoc 2009;27(Suppl 1):54.

12. Kleindorfer D, Khoury J, Broderick JP, Rademacher E, Woo D, Flaherty ML, et al. Temporal trends in public awareness of stroke. Stroke 2009;40:2502-2506.

13. Song HY, Hur HK, Park S. Lifestyle Risk Factors and Awareness of Stroke among Adults in Urban Areas. Korean J Health Promot 2012;12:47-57.

14. Kim YS, Park SS, Bae HJ, Cho AH, Cho YJ, Han MK, et al. Stroke awareness decreases prehospital delay after acute ischemic stroke in Korea. BMC Neurol 2011;11:2.

15. Korean Health Insurance Review \& Assessment Service. Report of Assessment for Quality of Acute Stroke Care in Korea 2005 .

16. Korean Health Insurance Review \& Assessment Service. Report of Assessment for Quality of Acute Stroke Care in Korea 2008.

17. Korean Health Insurance Review \& Assessment Service. Report of Assessment for Quality of Acute Stroke Care in Korea 2010.

18. Jung KH, Lee SH, Kim BJ, Yu KH, Hong KS, Lee BC, et al. Secular trends in ischemic stroke characteristics in a rapidly developed country: results from the Korean Stroke Registry Study (secular trends in Korean stroke). Circ Cardiovasc Qual Outcomes 2012;5:327-334.

19. Ryu JY, Eo EK, Kim YJ, Jung KY. Factors Associated with Delayed Arrival at the Hospital in Cases of Acute Stroke. J Korean Soc Emerg Med 2000;11:296-304.

20. Lee SH, Park YS, Chung SP, Park IC, Chung HS, Choi SH, et al. Factors in Delayed Arrival at the Emergency Department in Patients with Suspected Acute Stroke. J Korean Soc Emerg Med 2006; 17:431-437.

21. Hur JW, Jo IJ, Sim MS, Song HG. Factors Influencing Prehospital and Inhospital Time Delays for Ischemic Stroke Patients. J Korean Soc Emerg Med 2011;22:193-199.

22. Kim SK, Lee SY, Bae HJ, Lee YS, Kim SY, Kang MJ, et al. Prehospital notification reduced the door-to-needle time for iv t-PA in acute ischaemic stroke. Eur J Neurol 2009;16:13311335.

23. Kidwell CS, Saver JL, Schubert GB, Eckstein M, Starkman S. Design and retrospective analysis of the Los Angeles Prehospital Stroke Screen (LAPSS). Prehosp Emerg Care 1998;2:267- 
273.

24. Kothari RU, Pancioli A, Liu T, Brott T, Broderick J. Cincinnati Prehospital Stroke Scale: reproducibility and validity. Ann Emerg Med 1999;33:373-378.

25. Fonarow GC, Smith EE, Saver JL, Reeves MJ, Bhatt DL, GrauSepulveda MV, et al. Timeliness of Tissue-Type Plasminogen Activator Therapy in Acute Ischemic Stroke. Circulation 2011; 123:750-758.

26. Heo JH, Kim YD, Nam HS, Hong KS, Ahn SH, Cho HJ, et al. A computerized in-hospital alert system for thrombolysis in acute stroke. Stroke 2010;41:1978-1983.

27. Nam HS, Han SW, Ahn SH, Lee JY, Choi HY, Park IC, et al. Improved time intervals by implementation of computerized physician order entry-based stroke team approach. Cerebrovasc Dis 2007;23:289-293.

28. Choi HY, Cha MJ, Nam HS, Kim YD, Hong KS, Heo JH. Stroke units and stroke care services in Korea. Int J Stroke 2012;7:336-340.

29. Korean Health Insurance Review \& Assessment Service. Comprehensive Quality Report of National Health Insurance 2010. 